\title{
NON-LOCAL GEL'FAND PROBLEM IN HIGHER DIMENSIONS
}

\author{
TOSIYA MIYASITA and TAKASHI SUZUKI \\ Division of Mathematical Science \\ Graduate School of Engineering Science \\ Osaka University, Osaka 650-8531, Japan \\ E-mail: \{miyasita,suzuki\}@sigmath.es.osaka-u.ac.jp
}

\begin{abstract}
The non-local Gel'fand problem, $\Delta v+\lambda e^{v} / \int_{\Omega} e^{v} d x=0$ with Dirichlet boundary condition, is studied on an $n$-dimensional bounded domain $\Omega$. If it is star-shaped, then we have an upper bound of $\lambda$ for the existence of the solution. We also have infinitely many bendings in $\lambda$ of the connected component of the solution set in $\lambda, v$ if $\Omega$ is a ball and $3 \leq n \leq 9$.
\end{abstract}

1. Introduction. The purpose of the present paper is to study the Gel'fand problem with non-local term,

$$
\begin{cases}-\Delta v=\lambda \frac{e^{v}}{\int_{\Omega} e^{v} d x} & \text { in } \Omega, \\ v=0 & \text { on } \partial \Omega,\end{cases}
$$

where $\lambda$ is a positive constant and $\Omega$ is a bounded domain in $\mathbf{R}^{n}$ with smooth boundary $\partial \Omega$. Actually, the usual Gel'fand problem, in the theories of thermonic emission ([12]), isothermal gas sphere $([9])$, and gas combustion $([3])$, is formulated as the nonlinear eigenvalue problem

$$
\begin{cases}-\Delta v=\sigma e^{v} & \text { in } \Omega, \\ v=0 & \text { on } \partial \Omega,\end{cases}
$$

with a constant $\sigma>0$.

Problems (1) and (2) are equivalent through the relation

$$
\sigma=\lambda / \int_{\Omega} e^{v} d x
$$

and hence some features of the solution set

$$
\mathcal{C}=\{(\lambda, v) \mid v=v(x) \text { is a classical solution to (1) for } \lambda>0\}
$$

2000 Mathematics Subject Classification: 35J60, 35P30, 35J20.

The paper is in final form and no version of it will be published elsewhere. 
resemble those of the solution set for $(2)$, denoted by $\mathcal{S}$. For example, if $\Omega$ is a ball with $n \geq 3$, then $\mathcal{S}$ is a one-dimensional open manifold with the endpoints in $(\sigma, v)=(0,0)$ and $(\sigma, v)=(2(n-2), 2 \log (1 /|x|))$, respectively, the latter being a weak solution to $(2)$. This structure is kept by $\mathcal{C}$, just replacing the endpoints as $(\lambda, v)=(0,0)$ and

$$
(\lambda, v(x))=\left(2 \omega_{n}, 2 \log \frac{1}{|x|}\right),
$$

where $\omega_{n}$ denotes the $(n-1)$ dimensional volume of the surface of the unit ball in $\mathbf{R}^{n}$. Thus, $\mathcal{C}$ is still a one-dimensional open manifold. However, we know much more on $\mathcal{S}$ in this case, such as, infinitely many bendings with respect to $\sigma$ around $\sigma=2(n-2)$ with the radial Morse indices increasing by one for $3 \leq n \leq 9$, no bending in contrast for $n \geq 10$, and so forth ([12], [14], [23]), and it is not obvious that those structures are kept by (1). This is actually the case, as one of the results obtained in this paper assures.

In the case of $n=2$, we have several results on (1). In fact, it describes the equilibrium state of the mean field of many point vortices of the perfect fluid in Onsager's formulation ([7], [8], [17]). Mathematically, there are quantized blowup mechanisms for the family of solutions ([21], [2]), uniqueness of the solution in $0<\lambda<8 \pi$ for a simply connected domain ([28]), and calculation of the total degree for $\lambda \notin 8 \pi \mathcal{N}$ ([10]). Relative to this case is the mean field equation on Riemannian surfaces, and it has the origin in self-dual gauge theories, in geometry, and in mathematical biology ([32], [34], [1], [27]).

Recently, it is recognized that the equilibrium state of self-interacting particles is formulated as a non-local elliptic eigenvalue problem, and this is the reason why we here study the higher dimensional case of (1). We refer to [5], [29] as a typical example of such a theory, where the mean field of many self-gravitating particles is coupled with the temperature. If the temperature is put to be a constant, then it is reduced to (1). We also mention that the equilibrium of a simplified Keller-Segel system [16] for the chemotactic aggregation of cellular slime molds is similarly reduced to (1).

In fact, that system is given, for instance, by

$$
\begin{cases}\varepsilon u_{t}=\nabla \cdot(\nabla u-u \nabla v) & \text { in } \Omega \times(0, T) \\ \tau v_{t}=\Delta v+u & \text { in } \Omega \times(0, T) \\ \frac{\partial u}{\partial \nu}-u \frac{\partial v}{\partial \nu}=v=0 & \text { on } \partial \Omega \times(0, T) \\ \left.u\right|_{t=0}=u_{0}(x) \geq 0 & \text { in } \Omega \\ \left.v\right|_{t=0}=v_{0}(x) & \text { in } \Omega,\end{cases}
$$

where $\Omega$ is a bounded domain with smooth boundary $\partial \Omega, \tau, \varepsilon$ are positive constants, and $\nu$ is the outer unit normal vector respectively. Among many important profiles of the solution, we have $u=u(x, t) \geq 0$ and

$$
\varepsilon \frac{d}{d t} \int_{\Omega} u d x=\int_{\Omega} \nabla \cdot(\nabla u-u \nabla v) d x=0,
$$

which implies the total mass conservation, $\|u(\cdot, t)\|_{1}=\left\|u_{0}\right\|_{1}$. Here and henceforth, $\|\cdot\|_{p}$ denotes the standard $L^{p}$ norm. There is also a Lyapunov function in this system, which is nothing but the free energy physically, so that we have

$$
\varepsilon \frac{d}{d t} W(u, v)+\tau \varepsilon\left\|v_{t}\right\|_{2}^{2}+\int_{\Omega} u|\nabla(\log u-v)|^{2} d x=0
$$


for

$$
W(u, v)=\int_{\Omega}\left(u(\log u-1)-u v+\frac{1}{2}|\nabla v|^{2}\right) d x .
$$

This means that the $\omega$-limit set of the global in time solution is contained in the set of stationary solutions, with the $L^{1}$ norm of $u=u(x)$ prescribed by

$$
\|u\|_{1}=\lambda
$$

for $\lambda=\left\|u_{0}\right\|_{1}$. This status is actually realized by (1), as we have

$$
\log u-v=\log \sigma
$$

from (5) with $u, v$ independent of $t$, where the unknown constant $\sigma>0$ is prescribed as

$$
\sigma=\lambda / \int_{\Omega} e^{v} d x
$$

from (6).

To state the result, we take the section of $\mathcal{C}$ cut by $\lambda>0$ :

$$
\mathcal{C}^{\lambda}=\left\{v \in C^{2}(\Omega) \cap C(\bar{\Omega}) \mid v=v(x) \text { solves }(1)\right\} .
$$

The first theorem is concerned with the star-shaped domain, so that $x \cdot \nu>0$ holds for each $x \in \partial \Omega$.

THEOREM 1. If $\Omega$ is star-shaped, then there exists a positive number $\bar{\lambda}$ such that $\mathcal{C}^{\lambda}$ is nonempty for $\lambda<\bar{\lambda}$ and empty for $\lambda>\bar{\lambda}$. Moreover, $\mathcal{C}_{0}$ is unbounded in the $\lambda, v$ plane, and $\sharp \mathcal{C}^{\lambda}=1$ for $0<\lambda \ll 1$, where $\mathcal{C}_{0}$ stands for the connected component of $\mathcal{C}$ satisfying $(0,0) \in \overline{\mathcal{C}_{0}}$.

The second theorem concerns the ball case, where a similar result for (2) is given.

Theorem 2. If $\Omega$ is the unit ball $B=\left\{x \in \mathbf{R}^{n}|| x \mid<1\right\}$ and $3 \leq n \leq 9$, then $\mathcal{C}$ bends infinitely many times. On the other hand, no bending occurs in the case of $n \geq 10$.

As noted before, in the ball case $\mathcal{C}$ is a one-dimensional open manifold parametrized as

$$
\mathcal{C}=\{(\lambda(s), v(\cdot, s)) \mid 0<s<+\infty\}
$$

with the endpoints $(0,0)$ and the weak solution $(3)$. Namely,

$$
\lim _{s \downarrow 0}(\lambda(s), v(\cdot, s))=(0,0)
$$

and

$$
\lim _{s \uparrow+\infty}(\lambda(s), v(\cdot, s))=\left(2 \omega_{n}, 2 \log \frac{1}{|x|}\right)
$$

in $\mathbf{R} \times C(\bar{B})$ and $\mathbf{R} \times W^{2, p}(B)$ for $p \in[1, n / 2)$, respectively, where $\omega_{n}$ denotes the $(n-1)$ dimensional volume of the unit ball in $\mathbf{R}^{n}$. The above theorem says that if $3 \leq n \leq 9$, then there is a sequence $0<s_{1}<s_{2}<\cdots<s_{k}<\cdots$ such that $s \in\left[s_{2 k-1}, s_{2 k}\right] \mapsto \lambda(s)$ and $s \in\left[s_{2 k}, s_{2 k+1}\right] \mapsto \lambda(s)$ decreasing and increasing, respectively, and

$$
\begin{aligned}
\lambda\left(s_{2}\right)<\lambda\left(s_{4}\right) & <\cdots<\lambda\left(s_{2 k}\right)<\lambda\left(s_{2 k+2}\right)<\cdots<2 \omega_{n} \\
& <\cdots<\lambda\left(s_{2 k+1}\right)<\lambda\left(s_{2 k-1}\right)<\cdots<\lambda\left(s_{3}\right)<\lambda\left(s_{1}\right) .
\end{aligned}
$$


In particular, there are infinitely many solutions to (1) for $\lambda=2 \omega_{n}$. If $n \geq 10$, on the other hand, $s \in[0, \infty) \mapsto \lambda(s)$ is increasing, and each $\lambda \in\left(0,2 \omega_{n}\right)$ yields a unique solution to $(1)$.

We also have the following property in this case. Namely, given $(\lambda, v) \in \mathcal{C}$, the linearized eigenvalue problem is described as

$$
\begin{cases}-\Delta \phi-\lambda \frac{e^{v}}{\int_{\Omega} e^{v} d x} \phi+\lambda \frac{\int_{\Omega} e^{v} \phi d x}{\left(\int_{\Omega} e^{v} d x\right)^{2}} e^{v}=\mu \phi & \text { in } \Omega, \\ \phi=0 & \text { on } \partial \Omega .\end{cases}
$$

Then, the Morse index $i=i(\lambda, v)$ and the radial Morse index $i_{R}=i_{R}(\lambda, v)$ denote the number of negative eigenvalues and of radially symmetric eigenfunctions, respectively.

THEOREM 3. If $\Omega$ is a ball, then always $i=i_{R}$, and this index increases by one at each bending point.

The above theorem says that if $3 \leq n \leq 9$, then $i=i_{R}=k$ holds on the $\operatorname{arc} T_{k} T_{k+1}$ of $\mathcal{C}$, where $T_{k}=\left(\lambda\left(s_{k}\right), v\left(s_{k}\right)\right)$ for $k=0,1, \cdots$ with $s_{0}=0$. If $n \geq 10$, then on the other hand, always $i=i_{R}=0$.

For the annulus domain

$$
A=\left\{x \in \mathbf{R}^{n}|a<| x \mid<1\right\}
$$

with $a \in(0,1)$, we have some results for (2). From them, we see that the total set of radially symmetric solutions to $(1)$, denoted by $\mathcal{C}_{r}$, forms a one-dimensional open manifold with the trivial solution $(\lambda, v)=(0,0)$ as an endpoint, taking the entire blowup at the other endpoint $([22],[23])$. Thus, it is parametrized as $\mathcal{C}_{r}=\{(\lambda(s), v(\cdot, s)) \mid 0<s<+\infty\}$ such that $\lim _{s \downarrow 0}(\lambda(s), v(s))=(0,0)$ in $\mathbf{R} \times C(\bar{A})$, while $\lim _{s \uparrow+\infty} v(x, s)=+\infty$ for each $x \in A$. Furthermore, $\mathcal{C}_{r}$ bifurcates non-radially symmetric solutions infinitely many times. For this case, we also know that the number of bendings with respect to $\sigma$ of $\mathcal{S}$, the total set of solutions $(\sigma, v)$ to $(2)$, tends to infinity as $a \downarrow 0$. However, not much is known for (1) in this case, and even the behavior of $\lambda(s)$ as $s \rightarrow+\infty$ is open.

This paper is composed of four sections. In $\S 2$, we treat the star-shaped domain and prove Theorem 1. Next, we study the linearized spectrum concerning (1). This is different from that of (2), and $\S 3$ is devoted to its description. Section 4 is concerned with the ball case and Theorems 2 and 3 are proven there.

2. Star-shaped domain. Throughout the present section, $\Omega$ denotes the general starshaped domain with respect to the origin in $\mathbf{R}^{n}, n \geq 3$, with the smooth boundary $\partial \Omega$, and $\nu$ stands for the outer unit normal vector.

Proof of Theorem 1. It is proven in McGough [19] that the star-shaped $\Omega$ has $\tilde{\sigma}>0$ such that the solution $v=v(x)$ to (2) is unique for $0<\sigma<\tilde{\sigma}$. However, any solution $v=v(x)$ to (1) solves (2) with

$$
\sigma=\frac{\lambda}{\int_{\Omega} e^{v} d x} \leq \frac{\lambda}{|\Omega|}
$$

because of its positivity, where $|\Omega|$ denotes the volume of $\Omega$. Therefore, the solution to (1) is unique for $0<\lambda<\tilde{\lambda}=\tilde{\sigma}|\Omega|$, and the last part of the theorem is proven. 
The first part is already shown in [4], but we shall provide the proof for completeness. Actually, we make use of the Pohozaev identity [24] valid for (2)

$$
n \sigma \int_{\Omega}\left(e^{v}-1\right) d x+\frac{2-n}{2} \sigma \int_{\Omega} e^{v} v d x=\frac{1}{2} \int_{\partial \Omega}\left(\frac{\partial v}{\partial \nu}\right)^{2} d s .
$$

If $v=v(x)$ is a solution to (1), then the above equality is valid with $\sigma=\lambda / \int_{\Omega} e^{v} d x$, and hence it follows that

$$
\frac{1}{2} \int_{\partial \Omega}\left(\frac{\partial v}{\partial \nu}\right)^{2}(x \cdot \nu) d s \leq n \lambda
$$

On the other hand, we have

$$
\lambda=\int_{\Omega}(-\Delta v) d x=\int_{\partial \Omega}\left(-\frac{\partial v}{\partial \nu}\right) d s
$$

and therefore we obtain

$$
\lambda^{2} \leq \int_{\partial \Omega}\left(\frac{\partial v}{\partial \nu}\right)^{2}(x \cdot \nu) d s \cdot \int_{\partial \Omega} \frac{d s}{x \cdot \nu} \leq 2 n \lambda \int_{\partial \Omega} \frac{d s}{x \cdot \nu} .
$$

This gives the upper bound of $\lambda$.

Unboundedness of the component $\mathcal{C}_{0}$ follows from the standard degree argument similarly to [26], using the existence of the upper bound $\bar{\lambda}$ and the fact $\mu_{1}(\lambda, v)>0$ for $(\lambda, v) \in \underline{\mathcal{C}}$, where $\underline{\mathcal{C}}$ is some one dimensional manifold contained in $\mathcal{C}$ starting from $(\lambda, v)=(0,0)$.

3. Spectral property. The main difficulty to (1), in contrast with (2), is the lack of comparison principle. For example, existence of the minimal solution is not assured for given $\lambda$. Also, linearized stability changes the meaning, as the linearized eigenvalue problem is realized as $(8)$.

However, the first eigenvalue, denoted by $\mu_{1}(\lambda, v)$, is positive around the trivial solution $(\lambda, v)=(0,0)$ similarly to $(2)$. Therefore, it generates a branch in $\mathcal{C}$. This branch continues as far as $\mu_{1}(\lambda, v)>0$, and because we have an upper bound of $\lambda$ for $\mathcal{C}^{\lambda} \neq \emptyset$ to hold if $\Omega$ is star-shaped, only two possibilities arise then. That is, there is a one-dimensional manifold contained in $\mathcal{C}$ starting from $(\lambda, v)=(0,0)$ denoted by

$$
\underline{\mathcal{C}}=\left\{(\lambda(s), v(\cdot, s)) \mid 0<s<s_{0}\right\},
$$

and either $\lim _{s \rightarrow s_{0}}(\lambda(s), v(\cdot, s))=\left(\lambda^{*}, v^{*}\right) \in \mathcal{C}$ exists in $\mathbf{R} \times C(\bar{\Omega})$ with

$$
\mu_{1}\left(\lambda^{*}, v^{*}\right)=0,
$$

or else $\lim _{\sup _{s \rightarrow s_{0}}}\|v(\cdot, s)\|_{\infty}=+\infty$.

For simplicity, we say that $\underline{\mathcal{C}}$ is closed and open in the former and the latter cases, respectively. Those alternatives hold as far as there is an upper bound of $\lambda$ for the existence of the solution to (1). Even if this not the case, the connected component $\mathcal{C}_{0}$ mentioned in Theorem 1 contains this $\underline{\mathcal{C}}$.

An analogous situation occurs for $(2)([11],[6])$. It is known that the branch of minimal solutions is closed for $3 \leq n \leq 9$ and bends at $\sigma=\bar{\sigma}$, the upper bound of $\sigma$ for the existence of the solution. Even in the general case, we have uniqueness of the weak solution at $\sigma=\bar{\sigma}$. Unfortunately, we do not know whether those structures hold for $\underline{\mathcal{C}}$. 
In fact, linearized operator around $(\lambda, v) \in \mathcal{C}$, the left-hand side of $(8)$, is realized as a self-adjoint operator associated with the bilinear form

$$
\mathcal{A}(\phi, \phi)=\int_{\Omega}\left(|\nabla \phi|^{2}-u \phi^{2}\right) d x+\frac{1}{\lambda}\left(\int_{\Omega} u \phi d x\right)^{2}
$$

defined for $\phi \in H_{0}^{1}(\Omega)$, where $u=\lambda e^{v} / \int_{\Omega} e^{v} d x$. Therefore, if

$$
\underline{\mathcal{S}}=\left\{\left(\sigma, \underline{v}_{\sigma}\right) \mid 0<\sigma<\bar{\sigma}\right\}
$$

denotes the set of minimal solutions to (2) assured by [11] and

$$
\lambda(\sigma)=\sigma \int_{\Omega} e^{\underline{v}} \sigma x
$$

then $\left\{\left(\lambda(\sigma), \underline{v}_{\sigma}\right) \mid 0<\sigma<\bar{\sigma}\right\} \subset \underline{\mathcal{C}}$ because of the last term of the right-hand side of (9).

Letting the self-adjoint operator defined above to be $A$, we now describe its spectral properties. In fact, we have

$$
\mathcal{A}(\phi, \phi)=\int_{\Omega}|\nabla \phi|^{2} d x-\int_{\Omega} u\left(\phi-\frac{1}{\lambda} \int_{\Omega} u \phi d x\right)^{2} d x
$$

and now introduce the eigenvalue problem of finding $\phi \in H_{0}^{1}(\Omega)$ such that

$$
(\nabla \phi, \nabla \psi)=\nu(H \phi, H \psi)_{u} \quad \text { for any } \psi \in H_{0}^{1}(\Omega),
$$

where

$$
H \phi=\phi-\frac{1}{\lambda} \int_{\Omega} \phi u d x
$$

$\left(.,\right.$. ) stands for the standard $L^{2}$ inner product, and

$$
(\phi, \psi)_{u}=\int_{\Omega} \phi \psi u d x .
$$

Because $b(\phi, \psi)=(H \phi, H \psi)_{u}$ provides an inner product in $X=L^{2}(\Omega) / \mathbf{R}$, problem $(9)$ gives a complete orthogonal system in $V=H_{0}^{1}(\Omega)$ and $X$, the former provided with the Dirichlet norm. If $\left\{\nu_{k}\right\}_{k=1}^{\infty}$ denote its eigenvalues with $0<\nu_{1} \leq \nu_{2} \leq \cdots$, then $\nu_{k}>1$, $\nu_{k}=1, \nu_{k}<1$ are equivalent to $\mu_{k}>0, \mu_{k}=0, \mu_{k}<0$, where $\mu_{1} \leq \mu_{2} \leq \cdots$ denote the eigenvalues of $A$. This notation is compatible with $\mu_{1}=\mu_{1}(\lambda, v)$, and henceforth we sometimes write $\mu_{k}(\lambda, v)$ for this $\mu_{k}$.

Writing $\hat{\phi}=H \phi \in H_{c}^{1}(\Omega)$ in (10), we have

$$
(\nabla \hat{\phi}, \nabla \hat{\psi})=\nu(\hat{\phi}, \hat{\psi})_{u} \quad \text { for any } \hat{\psi} \in H_{c}^{1}(\Omega)
$$

where $H_{c}^{1}(\Omega)=H_{0}^{1}(\Omega) \oplus \mathbf{R}$. This eigenvalue problem provides a complete orthogonal system to $\hat{V}=H_{c}^{1}(\Omega)$ and $\hat{X}=L^{2}(\Omega)$, the latter provided with the norm $b(\cdot, \cdot)^{1 / 2}$. However, the Dirichlet norm is degenerate in rank one on $\hat{V}$, and actually the eigenvalues to (11) are given by $\left\{\nu_{k}\right\}_{k=0}^{\infty}$ with $\nu_{0}=0$. Thus, the first eigenfunction to (11) is a constant. To come back to (10) from (11) for $\nu>0$, we just put $\phi=\hat{\phi}-\left.\hat{\phi}\right|_{\partial \Omega}$. In fact, in this case we have $(\hat{\phi}, 1)_{u}=0$ and then (10) follows. On the other hand, problem (11) is realized as

$$
\begin{aligned}
& -\Delta \hat{\phi}=\nu u \hat{\phi} \text { in } \Omega, \\
& \hat{\phi}=\text { constant on } \partial \Omega, \\
& \int_{\partial \Omega} \frac{\partial \hat{\phi}}{\partial \nu} d s=0
\end{aligned}
$$


where $d s$ denotes the surface element of $\partial \Omega$. In this way, we get the following theorem. Equivalent facts are obtained by [33], [28], independently, and a unified description is given in [31].

Theorem 4. Given $(\lambda, v) \in \mathcal{C}$, the conditions $\mu_{1}(\lambda, v)>0, \mu_{1}(\lambda, v)=0, \mu_{1}(\lambda, v)<0$ are equivalent to $\nu_{1}>1, \nu_{1}=1, \nu_{1}<1$, respectively, where $\nu_{1}$ denotes the second eigenvalue of (12) characterized by

$$
\nu_{1}=\inf \left\{\|\nabla \psi\|_{2}^{2} \mid \psi \in H_{c}^{1}(\Omega), \quad \int_{\Omega} \psi u d x=0, \quad \int_{\Omega} \psi^{2} u d x=1\right\}
$$

for $u=\lambda e^{v} / \int_{\Omega} e^{v} d x$.

Generally, the second eigenfunction to (11) denoted by $\hat{\phi}_{1}$ has two nodal domains. However, $\phi_{1}=\hat{\phi}_{1}-\left.\hat{\phi}_{1}\right|_{\partial \Omega}$ may have constant sign. If this is the case, then we can repeat the argument of [11]. Namely, we get the following.

Proposition 1. If $\left(\lambda^{*}, v^{*}\right) \in \mathcal{C}$ satisfies $\mu_{2}\left(\lambda^{*}, v^{*}\right)>\mu_{1}\left(\lambda^{*}, v^{*}\right)=0$, with $\mu_{1}\left(\lambda^{*}, v^{*}\right)=0$ admitting the eigenfunction $\phi^{*}>0$, then $\mathcal{C}$ is locally a one-dimensional manifold around it, parametrized as

$$
\mathcal{C}^{*}=\{(\lambda(s), v(s))|| s \mid<\delta\}
$$

with $(\lambda(0), v(0))=\left(\lambda^{*}, v^{*}\right)$. Furthermore, $\mathcal{C}^{*}$ bends to the left with respect to $\lambda$ at $\left(\lambda^{*}, v^{*}\right)$, so that $\lambda(s)<\lambda^{*}$ for $0<|s|<\delta$ and the mappings $s \in(-\delta, 0] \mapsto \lambda(s)$ and $s \in[0, \delta) \mapsto$ $\lambda(s)$ are increasing and decreasing, respectively. Finally, $\mu_{1}(\lambda(s), v(s))$ changes sign at $s=0$, say, $\pm \mu_{1}(\lambda(s), v(s))>0$ according as $-\delta< \pm s<0$.

Proof. Given $\left(\lambda^{*}, v^{*}\right) \in \mathcal{C}$ with $\mu_{1}\left(\lambda^{*}, v^{*}\right)=0$, let the linearized operator, the left-hand side of $(8)$ with $(\lambda, v)=\left(\lambda^{*}, v^{*}\right)$ be $A^{*}$. Then, from the assumption we have $\operatorname{Ker}\left(A^{*}\right)=$ $\left\langle\phi^{*}\right\rangle$ with $\phi^{*}=\phi^{*}(x) \in H_{0}^{1}(\Omega) \backslash\{0\}$ positive in $\Omega$. Now, we take the nonlinear operator

$$
\Phi(s, \sigma, w)=\Delta\left(v^{*}+s \phi^{*}+w\right)+\left(\lambda^{*}+\sigma\right) \frac{e^{v^{*}+s \phi^{*}+w}}{\int_{\Omega} e^{v^{*}+s \phi^{*}+w} d x},
$$

defined for $s \in \mathbf{R}, \sigma \in \mathbf{R}$, and $w \in Y$, where

$$
Y=\left\{w \in C^{2}(\bar{\Omega})|w|_{\partial \Omega}=0, \quad \int_{\Omega} w \phi^{*} d x=0\right\} .
$$

It is obvious that $\Phi(0,0,0)=0$, and the linearized operator

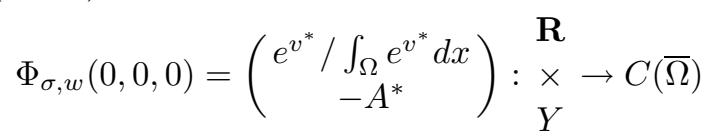

is an isomorphism by $\phi^{*}>0$. Because a classical solution to (1) near $\left(\lambda^{*}, v^{*}\right)$ is identified with a zero of $\Phi$, the implicit function theorem then guarantees a $C^{2}$-family $\{(\lambda(s), v(s)) \mid$ $\left.|s|<s_{0}\right\}$ of classical solutions satisfying $(\lambda(0), v(0))=\left(\lambda^{*}, v^{*}\right)$, where $s_{0}>0$. It also follows from the standard perturbation theory (Theorems VI.5.10 and VII.1.14 of [15]) that the linearized operator around this $(\lambda(s), v(s))$ has the simple eigenvalue $\mu(s)$ and the eigenfunction $\phi(s)$ with $C^{2}$ dependence in $s$ such that $(\mu(0), \phi(0))=\left(0, \phi^{*}\right)$ so that (8) is valid for

$$
(\lambda, v, \mu, \phi)=(\lambda(s), v(s), \mu(s), \phi(s))
$$

with $|s|<s_{0}$. 
Differentiating with respect to $s$, we have from (1)

$$
\begin{aligned}
& \Delta \dot{v}+\dot{\lambda} \frac{e^{v}}{\int_{\Omega} e^{v} d x}+\lambda \frac{e^{v}}{\int_{\Omega} e^{v} d x} \dot{v}-\lambda \frac{\int_{\Omega} e^{v} \dot{v} d x}{\left(\int_{\Omega} e^{v} d x\right)^{2}} e^{v}=0 \quad \text { in } \Omega, \\
& \dot{v}=0
\end{aligned}
$$

Then, subtracting (8) from (13) with $s=0$, multiplied by $\dot{v}$ and $\phi^{*}$, respectively, we get

$$
\dot{\lambda}(0) \frac{\int_{\Omega} e^{v^{*}} \phi^{*} d x}{\int_{\Omega} e^{v^{*}} d x}=0
$$

and hence $\dot{\lambda}(0)=0$ holds true. This implies $\dot{v}(0) \in \operatorname{Ker} A^{*}$ by (13), and we can assume

$$
\dot{v}(0)=\phi^{*}
$$

without loss of generality, because $(\dot{\lambda}(0), \dot{v}(0))$ does not vanish by the implicit function theorem.

Differentiating (13) once more and putting $s=0$, we have

$$
\begin{aligned}
& \Delta \ddot{v}+\ddot{\lambda} \frac{e^{v}}{\int_{\Omega} e^{v} d x}+\lambda \frac{e^{v} \phi^{* 2}}{\int_{\Omega} e^{v} d x}+\lambda \frac{e^{v} \ddot{v}}{\int_{\Omega} e^{v} d x}-2 \lambda \frac{\int_{\Omega} e^{v} \phi^{*} d x}{\left(\int_{\Omega} e^{v} d x\right)^{2}} e^{v} \phi^{*} \\
& -\lambda \frac{\int_{\Omega} e^{v} \phi^{* 2} d x}{\left(\int_{\Omega} e^{v} d x\right)^{2}} e^{v}-\lambda \frac{\int_{\Omega} e^{v} \ddot{v} d x}{\left(\int_{\Omega} e^{v} d x\right)^{2}} e^{v}+2 \lambda \frac{\left(\int_{\Omega} e^{v} \phi^{*} d x\right)^{2}}{\left(\int_{\Omega} e^{v} d x\right)^{3}} e^{v}=0 \quad \text { in } \Omega
\end{aligned}
$$

with $\ddot{v}=0$ on $\partial \Omega$. Then, integrating (14) multiplied by $\phi^{*}$, we obtain

$$
\ddot{\lambda}(0) \frac{\int_{\Omega} e^{v^{*}} \phi^{*} d x}{\int_{\Omega} e^{v^{*}} d x}=\lambda^{*}\left\{3 \frac{\int_{\Omega} e^{v^{*}} \phi^{*} d x \int_{\Omega} e^{v^{*}} \phi^{* 2} d x}{\left(\int_{\Omega} e^{v^{*}} d x\right)^{2}}-2 \frac{\left(\int_{\Omega} e^{v^{*}} \phi^{*} d x\right)^{3}}{\left(\int_{\Omega} e^{v^{*}} d x\right)^{3}}-\frac{\int_{\Omega} e^{v^{*}} \phi^{* 3} d x}{\int_{\Omega} e^{v^{*}} d x}\right\} .
$$

Letting $\frac{e^{v^{*}} d x}{\int_{\Omega} e^{v^{*}} d x}=d \mu$, we have

$$
\begin{aligned}
\frac{\lambda \ddot{(0)}}{\lambda^{*}} \int_{\Omega} \phi^{*} d \mu & =3 \int_{\Omega} \phi^{*} d \mu \int_{\Omega} \phi^{* 2} d \mu-2\left(\int_{\Omega} \phi^{*} d \mu\right)^{3}-\int_{\Omega} \phi^{* 3} d \mu \\
& =3 \int_{\Omega} \phi^{*} d \mu \cdot\left\{\int_{\Omega} \phi^{* 2} d \mu-\left(\int_{\Omega} \phi^{*} d \mu\right)^{2}\right\}+\left(\int_{\Omega} \phi^{*} d \mu\right)^{3}-\int_{\Omega} \phi^{* 3} d \mu \leq 0
\end{aligned}
$$

with the equality only when $\phi^{*}$ is a constant. This is impossible, and we get $\ddot{\lambda}(0)<0$.

To complete the proof, we differentiate (8) and obtain

$$
\begin{aligned}
& \Delta \dot{\phi}+\lambda \frac{e^{v} \phi^{* 2}}{\int_{\Omega} e^{v} d x}-\lambda \frac{\int_{\Omega} e^{v} \phi^{*} d x}{\left(\int_{\Omega} e^{v} d x\right)^{2}} e^{v} \phi^{*}+\lambda \frac{e^{v} \dot{\phi}}{\int_{\Omega} e^{v} d x}-\lambda \frac{\int_{\Omega} e^{v} \phi^{* 2} d x}{\left(\int_{\Omega} e^{v} d x\right)^{2}} e^{v} \\
& -\lambda \frac{\int_{\Omega} e^{v} \dot{\phi} d x}{\left(\int_{\Omega} e^{v} d x\right)^{2}} e^{v}+2 \lambda \frac{\left(\int_{\Omega} e^{v} \phi^{*} d x\right)^{2}}{\left(\int_{\Omega} e^{v} d x\right)^{3}} e^{v}-\lambda \frac{\int_{\Omega} e^{v} \phi^{*} d x}{\left(\int_{\Omega} e^{v} d x\right)^{2}} e^{v} \phi^{*}=-\dot{\mu} \phi^{*}
\end{aligned}
$$

in $\Omega$ with $\dot{\phi}=0$ on $\partial \Omega$ by putting $s=0$. Then, from the Fredholm alternative we have

$$
-\dot{\mu}(0) \frac{\left\|\phi^{*}\right\|_{2}^{2}}{\lambda^{*}}=\int_{\Omega} \phi^{* 3} d \mu-3 \int_{\Omega} \phi^{*} d \mu \cdot \int_{\Omega} \phi^{* 2} d \mu+2\left(\int_{\Omega} \phi^{*} d \mu\right)^{3},
$$

similarly. The proof is complete. 
For $(2), \underline{\mathcal{S}}$, the branch of minimal solutions is bounded in $\mathbf{R} \times C(\bar{\Omega})$ in the case of $3 \leq n \leq 9$ ([11]). However, such a result is not known for $\underline{\mathcal{C}}$, because of the last term of the right-hand side of (9) and the non-local term of (1). On the other hand, if there is a weak or classical solution to (2) at the other endpoint then $(\sigma, v)=(0,0)$ for the general star-shaped domain $([20])$. This result is valid for $\underline{\mathcal{C}}$ because the non-local term of (1) is controlled by

$$
\int_{\Omega} e^{v} d x=\frac{\lambda}{\sigma}
$$

where $0<\sigma \ll 1$ admits a unique classical solution $v=v(x)$ to (2). In the proof of Proposition 1, the transversality condition

$$
\int_{\Omega} e^{v^{*}} \phi^{*} d x \neq 0
$$

plays a role. In terms of $\hat{\phi}^{*}$, the second eigenfunction of (12) for

$$
u=\lambda e^{v^{*}} / \int_{\Omega} e^{v^{*}} d x
$$

this means that $\hat{\phi} \neq 0$ on $\partial \Omega$. Namely, the transversality condition (15) is violated at $(\lambda, v)=\left(\lambda^{*}, v^{*}\right)$ if and only if this $\hat{\phi}^{*}$ has two nodal domains, with closures not contained in $\Omega$, and $\hat{\phi}^{*}=0$ on $\partial \Omega$. In other words, the second eigenfunction to

$$
-\Delta \phi=\nu u \phi \quad \text { in } \Omega, \quad \phi=0 \quad \text { on } \partial \Omega
$$

satisfies

$$
\int_{\partial \Omega} \frac{\partial \phi}{\partial \nu} d s=0
$$

4. Ball case. In this section, we assume $\Omega=B$, where $B=\left\{x \in \mathbf{R}^{n}|| x \mid<1\right\}$. First, we give the following.

Proof of Theorem 2. First, any solution to (1) is radially symmetric ([13]), so that we have

$$
v=v(r) \quad \text { for } \quad r=|x| .
$$

In other words, (2) induces

$$
\begin{aligned}
& \left(r^{n-1} v^{\prime}\right)^{\prime}+\sigma r^{n-1} e^{v}=0 \quad \text { for } r>0, \\
& v(0)=A, \quad v^{\prime}(0)=0,
\end{aligned}
$$

for $\sigma=\lambda / \int_{\Omega} e^{v} d x$ in this case, and then the standard Emden transformation

$$
v(r)=w(t)-2 t+A, \quad r=\left\{\frac{2(n-2)}{\sigma e^{A}}\right\}^{\frac{1}{2}} e^{t},
$$

reduces problem (17) to the autonomous ordinary differential equation

$$
\begin{aligned}
& \ddot{w}+(n-2) \dot{w}+2(n-2)\left(e^{w}-1\right)=0, \\
& \lim _{t \rightarrow-\infty}(w(t)-2 t)=\lim _{t \rightarrow-\infty} e^{-t}(\dot{w}(t)-2)=0 .
\end{aligned}
$$

The unique solution to this problem exists globally in time, and the orbit $\mathcal{O}=\{(w(t), \dot{w}(t)) \mid$ $t \in \mathbf{R}\}$ starts at $t=-\infty$ along and below the line $\dot{w}=2$ with $w=-\infty$, and approaches 
the origin $(0,0)$ as $t \rightarrow+\infty$. If $3 \leq n \leq 9$, it spirals clockwise in $\{(w, \dot{w}) \mid \dot{w}<2\}$, crosses infinitely many times the $\dot{w}$ - and $w$-axes alternately, while for $n \geq 10$ it stays forever in $\{(w, \dot{w}) \mid w<0, \dot{w}<2\}$.

Conversely, given $(w(\tau), \dot{w}(\tau)) \in \mathcal{O}$, we can take $v=v(r)$ through the reverse transformation, which satisfies (2) if $v(1)=0$, or equivalently,

$$
w(\tau)-2 \tau+A=0
$$

and

$$
\left\{\frac{2(n-2)}{\sigma e^{A}}\right\}^{1 / 2} e^{\tau}=1 .
$$

Thus, $\sigma$ is also recovered by $w(\tau)$ as

$$
\sigma=2(n-2) e^{2 \tau-A}=2(n-2) e^{w(\tau)} .
$$

From this well-known situation ([14], [22], [30]), the total set $\mathcal{S}$ of the solutions $(\sigma, v)$ to (2) is homeomorphic to $\mathcal{O}$, through the relation (18) with the constants $A, \sigma$ determined by (20), (21), respectively. This means also that $\mathcal{C}$ is homeomorphic to $\mathcal{O}$.

In fact, we have from (18) and (20) that

$$
\begin{aligned}
v(r) & =w(t)-2 t+(2 \tau-w(\tau)) \\
& =w(\tau+\log r)-2(\tau+\log r)+2 \tau-w(\tau) \\
& =w(\log r+\tau)-w(\tau)-2 \log r
\end{aligned}
$$

and hence relation (7) holds true. Thus, the theorem is a direct consequence of the following identity:

$$
\lambda=\omega_{n}(2-\dot{w}(\tau)) .
$$

To prove this, we again make use of (22). In fact, we have

$$
v_{r}(1)=\dot{w}(\tau)-2
$$

and hence (23) follows from

$$
\lambda=\int_{B}(-\Delta v) d x=-\omega_{n} v_{r}(1) .
$$

The proof is complete.

Now, we give the following.

Proof of Theorem 3. Every solution to (1) is radially symmetric as in (16), and therefore, the eigenvalue problem $(8)$ is reduced to the case of

$$
\phi_{k}=\psi^{k}(r) e_{k}(\omega)
$$

of separation of variables, where $\omega=x / r$ and $\left\{e_{k}\right\}_{k=1}^{\infty}$ stands for the set of eigenfunctions of the Laplace-Beltrami operator on $\partial B$, denoted by $\Lambda$. In the case of $k \geq 2$, we have

$$
\int_{B} e^{v} \phi_{k} d x=0
$$

and hence it follows that

$$
\left\{\begin{array}{l}
-\psi_{r r}^{k}-\frac{n-1}{r} \psi_{r}^{k}+\frac{\nu_{k}}{r^{2}} \psi^{k}-\lambda \frac{e^{v}}{\int_{B} e^{v} d x} \psi^{k}=\mu_{k} \psi^{k} \quad(0<r<1) \\
\psi_{r}^{k}(0)=\psi^{k}(1)=0
\end{array}\right.
$$


for $\mu=\mu_{k}$, where $\nu_{k}$ denotes the eigenvalue of $\Lambda$ corresponding to $e_{k}$ labeled as $\nu_{1}=0<$ $\nu_{2} \leq \nu_{3} \leq \cdots$.

Problem (24) is of the same form obtained for (2) under the same process of linearization and separation of variables. Therefore, we have $\mu<0$ in this case by the argument of [18]. In fact, we have for $0 \leq r_{0}<r_{1} \leq 1$ that

$\left[\psi_{r}^{k} v_{r} r^{n-1}-\psi^{k} v_{r r} r^{n-1}\right]_{r=r_{0}}^{r=r_{1}}+\int_{r=r_{0}}^{r=r_{1}} \frac{n-1-\nu_{k}}{r^{2}} \psi^{k} v_{r} r^{n-1} d r=-\mu_{k} \int_{r=r_{0}}^{r=r_{1}} \psi^{k} v_{r} r^{n-1} d r$.

We take $r_{0}=0$ and the first zero of $\psi^{k}$ as $r_{1}$. Then, we have $\mu_{k}>0$ for $k>1$ because $\nu_{k} \geq n-1$ holds in this case. Thus, we have $i(\lambda, v)=i_{R}(\lambda, v)$ for $(\lambda, v) \in \mathcal{C}$.

Now, we turn to the movement of the radial Morse index, $i_{R}(\lambda, v)$. We note that $\mathcal{C}$ is homeomorphic to $\mathcal{O}$ by Theorem 2. Each point of $\mathcal{O}$ is given as $(w(\tau), \dot{w}(\tau))$ and hence $\mathcal{C}$ is parametrized by $\tau \in \mathbf{R}$, as $(v(\cdot, \tau), \lambda(\tau)) \in \mathcal{C}$ is realized as

$$
v(r, \tau)=w(\log r+\tau)-w(\tau)-2 \log r
$$

and

$$
\lambda(\tau)=\omega_{n}(2-\dot{w}(\tau)) .
$$

To make the description simple, henceforth the set of eigenvalues of the linearized operator around $(\lambda(\tau), v(\tau)) \in \mathcal{C}$ corresponding to radially symmetric eigenfunctions is denoted by $\sigma_{r}(\tau)=\left\{\mu_{k}^{r}(\tau)\right\}_{k=1}^{\infty}$. Any of them is simple and $\mu_{1}^{r}(s)<\mu_{2}^{r}(s)<\cdots$. Then, the standard bifurcation theorem from the critical point of odd multiplicity [25], [26] guarantees that if $0 \in \sigma_{r}(\tau)$ occurs for $(\lambda(\tau), v(\tau))$ not at the turning point of $\mathcal{C}$, then it is a bifurcation point for the problem

$$
-v_{r r}-\frac{n-1}{r} v_{r}=\lambda \frac{e^{v}}{\omega_{n} \int_{0}^{1} e^{v} r^{n-1} d r} \quad(0<r<1)
$$

with

$$
v_{r}(0)=v(1)=0
$$

or equivalently for (1). This is impossible from the geometric feature of $\mathcal{C}$ given in Theorem 2. Thus, we have $0 \notin \sigma_{r}(\tau)$ unless $(\lambda(\tau), v(\tau))$ is at the turning point of $\mathcal{C}$. On the other hand, $\mu_{1}^{r}(\tau)>0$ for $\tau \ll-1$, and therefore, $i_{R}(\lambda, v)=0$ for any $(\lambda, v) \in \mathcal{C}$ in the case of $n \geq 10$.

On the contrary, any turning point takes 0 in $\sigma_{r}(s)$ by the implicit function theorem, and we have actually infinitely many bending points in the other case of $3 \leq n \leq 9$, denoted by $T_{k}=\left(\lambda\left(\tau_{k}\right), v\left(\tau_{k}\right)\right) \in \mathcal{C}$ for $\tau_{1}<\tau_{2}<\cdots$. This means that each $k$ takes $\ell$ such that $\mu_{\ell}^{r}\left(\tau_{k}\right)=0$. Under those notations, we see that the assertion follows if

$$
\left.\dot{\mu}_{\ell}^{r}\right|_{\tau=\tau_{k}}<0
$$

is proven for any $k=1,2, \cdots$, where $\dot{\mu}$ stands for $d \mu / d \tau$. Now, we shall show that $(27)$ is actually true.

First, we have

$$
0=\dot{\lambda}\left(\tau_{k}\right)=-\omega_{n} \ddot{w}\left(\tau_{k}\right)
$$


because $T_{k}$ is the turning point of $\mathcal{C}$. Next, we have from $(2)$

$$
\begin{cases}\Delta \dot{v}+\dot{\lambda} \frac{e^{v}}{\int_{B} e^{v} d x}+\lambda \frac{e^{v} \dot{v}}{\int_{B} e^{v} d x}-\lambda \frac{\int_{B} e^{v} \dot{v} d x}{\left(\int_{B} e^{v} d x\right)^{2}} e^{v}=0 & \text { in } B \\ \dot{v}_{\tau}=0 & \text { on } \partial B\end{cases}
$$

and hence it follows that

$$
\begin{cases}\Delta \dot{v}_{k}+\lambda_{k} \frac{e^{v_{k}} \dot{v}_{k}}{\int_{B} e^{v_{k}} d x}-\lambda_{k} \frac{\int_{B} e^{v_{k}} \dot{v}_{k} d x}{\left(\int_{B} e^{v_{k}} d x\right)^{2}} e^{v_{k}}=0 & \text { in } B \\ \dot{v}_{k}=0 & \text { on } \partial B\end{cases}
$$

for $v_{k}=v\left(\cdot, \tau_{k}\right)$. We have

$$
\dot{v}(r, \tau)=\dot{w}(\log r+\tau)-\dot{w}(\tau) \not \equiv 0,
$$

and therefore, $\dot{v}_{k}$ is an eigenfunction to (8) corresponding to $\mu=\mu_{\ell}^{r}\left(\tau_{k}\right)=0$. Then, the standard perturbation theory guarantees the existence of $\phi=\phi(\cdot, \tau)$ and $\mu=\mu(\tau)$ satisfying $(8), \phi\left(\cdot, \tau_{k}\right)=\dot{v}_{k}$, and $\mu\left(\tau_{k}\right)=\mu_{\ell}^{r}\left(\tau_{k}\right)=0$.

We differentiate (29) and this with respect to $\tau$, and then put $\tau=\tau_{k}$. Then, by means of $\dot{\lambda}\left(\tau_{k}\right)=\mu\left(\tau_{k}\right)=0$, we obtain

$$
\begin{aligned}
& \Delta \ddot{v}+\ddot{\lambda} \frac{e^{v}}{\int_{B} e^{v} d x}+\lambda \frac{e^{v} \dot{v}^{2}}{\int_{B} e^{v} d x}+\lambda \frac{e^{v} \ddot{v}}{\int_{B} e^{v} d x} \\
& -\lambda \frac{\int_{B} e^{v} \dot{v} d x}{\left(\int_{B} e^{v} d x\right)^{2}} e^{v} \dot{v}-\lambda \frac{\int_{B} e^{v} \ddot{v} d x}{\left(\int_{B} e^{v} d x\right)^{2}} e^{v}-\lambda \frac{\int_{B} e^{v} \dot{v}^{2} d x}{\left(\int_{B} e^{v} d x\right)^{2}} e^{v} \\
& -\lambda \frac{\int_{B} e^{v} \dot{v} d x}{\left(\int_{B} e^{v} d x\right)^{2}} e^{v} \dot{v}+2 \lambda \frac{\left(\int_{B} e^{v} \dot{v} d x\right)^{2}}{\left(\int_{B} e^{v} d x\right)^{3}} e^{v}=0
\end{aligned}
$$

in $B$ with

$$
\ddot{v}=0 \quad \text { on } \quad \partial B
$$

and

$$
\begin{aligned}
& \Delta \dot{\phi}+\lambda \frac{e^{v} \dot{v} \phi}{\int_{B} e^{v} d x}-\lambda \frac{\int_{B} e^{v} \dot{v} d x}{\left(\int_{B} e^{v} d x\right)^{2}} e^{v} \phi+\lambda \frac{e^{v} \dot{\phi}}{\int_{B} e^{v} d x}-\lambda \frac{\int_{B} e^{v} \dot{v} \phi d x}{\left(\int_{B} e^{v} d x\right)^{2}} e^{v} \\
& -\lambda \frac{\int_{B} e^{v} \dot{\phi} d x}{\left(\int_{B} e^{v} d x\right)^{2}} e^{v}+2 \lambda \frac{\left(\int_{B} e^{v} \dot{v} d x\right)\left(\int_{B} e^{v} \phi d x\right)}{\left(\int_{B} e^{v} d x\right)^{3}} e^{v}-\lambda \frac{\int_{B} e^{v} \phi d x}{\left(\int_{B} e^{v} d x\right)^{2}} e^{v} \dot{v} \\
& =-\dot{\mu} \phi
\end{aligned}
$$

in $B$ with

$$
\dot{\phi}=0 \quad \text { on } \quad \partial B
$$

respectively, where $\lambda=\lambda\left(\tau_{k}\right), \dot{\lambda}=\dot{\lambda}\left(\tau_{k}\right), \dot{\mu}=\dot{\mu}\left(\tau_{k}\right), \ddot{\lambda}=\ddot{\lambda}\left(\tau_{k}\right), v=v\left(\cdot, \tau_{k}\right), \dot{v}=\dot{v}\left(\cdot, \tau_{k}\right)$, $\ddot{v}=\ddot{v}\left(\cdot, \tau_{k}\right), \phi=\phi\left(\cdot, \tau_{k}\right)$, and $\dot{\phi}=\dot{\phi}\left(\cdot, \tau_{k}\right)$. Multiplying those equalities by $\dot{v}=\phi$, we have

$$
\ddot{\lambda} \frac{\int_{B} e^{v} \dot{v} d x}{\int_{B} e^{v} d x}+\lambda \frac{\int_{B} e^{v} \dot{v}^{3} d x}{\int_{B} e^{v} d x}+2 \lambda \frac{\left(\int_{B} e^{v} \dot{v} d x\right)^{3}}{\left(\int_{B} e^{v} d x\right)^{3}}-3 \lambda \frac{\left(\int_{B} e^{v} \dot{v} d x\right)\left(\int_{B} e^{v} \dot{v}^{2} d x\right)}{\left(\int_{B} e^{v} d x\right)^{2}}=0
$$

and

$$
\lambda \frac{\int_{B} e^{v} \dot{v}^{3} d x}{\int_{B} e^{v} d x}+2 \lambda \frac{\left(\int_{B} e^{v} \dot{v} d x\right)^{3}}{\left(\int_{B} e^{v} d x\right)^{3}}-3 \lambda \frac{\left(\int_{B} e^{v} \dot{v} d x\right)\left(\int_{B} e^{v} \dot{v}^{2} d x\right)}{\left(\int_{B} e^{v} d x\right)^{2}}=-\dot{\mu} \int_{B} \dot{v}^{2} d x
$$


Therefore, it follows that

$$
\ddot{\lambda} \frac{\int_{B} e^{v} \dot{v} d x}{\int_{B} e^{v} d x}=\dot{\mu} \int_{B} \dot{v}^{2} d x .
$$

Here, we have from (26), (28), and (21) that

$$
\ddot{\lambda}=2(n-2) \omega_{n} e^{w} \dot{w}=\omega_{n} \dot{\sigma}
$$

where $w=w\left(\tau_{k}\right), \dot{w}=\dot{w}\left(\tau_{k}\right)$, and $\dot{\sigma}=\dot{\sigma}\left(\tau_{k}\right)$. Then, using

$$
\sigma=\frac{\lambda}{\int_{B} e^{v} d x}
$$

we get

$$
\dot{\sigma}=-\lambda \frac{\int_{B} e^{v} \dot{v} d x}{\left(\int_{B} e^{v} d x\right)^{2}}
$$

again by $\dot{\lambda}=\dot{\lambda}\left(\tau_{k}\right)=0$, and hence we obtain

$$
-\omega_{n} \lambda \frac{\left(\int_{B} e^{v} \dot{v} d x\right)^{2}}{\left(\int_{B} e^{v} d x\right)^{3}}=\dot{\mu} \int_{B} \dot{v}^{2} d x
$$

from (33). Thus, (27) is proven by

$$
\frac{d}{d \tau} \int_{B} e^{v} d x=\int_{B} e^{v} \dot{v} d x \neq 0 \quad \text { at } \quad \tau=\tau_{k} .
$$

However, this is equivalent to $\dot{\sigma} \neq 0$ there, because

$$
\int_{B} e^{v} d x=\frac{\lambda}{\sigma}
$$

and (28). Using (21), we get

$$
\dot{\sigma}=2(n-2) e^{w} \dot{w}
$$

at $\tau=\tau_{k}$, and the desired relation $\dot{\sigma}\left(\tau_{k}\right) \neq 0$ is proven by $\dot{w}\left(\tau_{k}\right) \neq 0$. This relation actually holds because $\ddot{w}\left(\tau_{k}\right)=0$ and $w=w(t)$ satisfies the autonomous system (19).

\section{References}

[1] T. Aubin, Some Nonlinear Problems in Riemannian Geometry, Springer, Berlin, 1998.

[2] S. Baraket and F. Pacard, Construction of singular limits for a semilinear elliptic equation in dimension 2, Calc. Var. 6 (1998), 1-38.

[3] J. Bebernes and D. Eberly, Mathematical Problems from Combustion Theory, Springer, New York, 1989.

[4] J. W. Bebernes and A. A. Lacey, Global existence and finite-time blow-up for a class of nonlocal parabolic problems, Adv. Diff. Equations 2 (1997), 927-953.

[5] P. Biler, J. Dolbeault, M. J. Esteban, P. A. Markowich, and T. Nadzieja, Steady states for Streater's energy-transport models of self-gravitating particles, in: Transport in Transition Regimes, N. Ben Abdallah, A. Arnold, P. Degond et al. (eds.), Springer IMA Volumes in Mathematics and Its Applications 135, 2003, 37-56.

[6] H. Brezis, T. Cazenave, Y. Martel, and R. Ramiandrisoa, Blowup for $u_{t}-\Delta u=g(u)$ revisited, Adv. Diff. Equations 1 (1996), 73-90. 
[7] E. Caglioti, P. L. Lions, C. Marchioro, and M. Pulvirenti, A special class of stationary flows for two-dimensional Euler equations: A statistical mechanics description, Comm. Math. Phys. 143 (1992), 501-525.

[8] - A special class of stationary flows for two-dimensional Euler equations: A statistical mechanics description, II, Comm. Math. Phys. 174 (1995), 229-260.

[9] S. Chandrasekhar, An Introduction to the Study of Stellar Structure, Dover, New York 1957.

[10] C.-C. Chen and C.-S. Lin, Topological degree for a mean field equation on Riemann surfaces, preprint.

[11] M. G. Crandall and P. H. Rabinowitz, Some continuation and variational methods for positive solutions of nonlinear elliptic eigenvalue problems, Arch. Rational Mech. Anal. 58 (1975), 207-218.

[12] I. M. Gel'fand, Some problems in the theory of quasilinear equations, Amer. Math. Soc. Transl. 29 (1963), 295-381.

[13] B. Gidas, W.-M. Ni, and L. Nirenberg, Symmetry and related properties via the maximal principle, Comm. Math. Phys. 68 (1979), 209-243.

[14] D. D. Joseph and T.S. Lundgren, Quasilinear Dirichlet problems driven by positive sources, Arch. Rational Mech. Anal. 49 (1973), 241-269.

[15] T. Kato, Perturbation Theory for Linear Operators, Springer, Berlin, 1966.

[16] E. F. Keller and L. A. Segel, Initiation of slime mold aggregation viewed as an instability, J. Theor. Biol 36 (1970), 399-415.

[17] M. K. H. Kiessling, Statistical mechanics of classical particles with logarithmic interactions, Comm. Pure Appl. Math. 46 (1993), 27-56.

[18] C.-S. Lin and W.-M. Ni, A counterexample to the nodal domain conjecture and a related semilinear equation, Proc. Amer. Math. Soc. 102 (1988), 271-277.

[19] J. McGough, On solution continua of supercritical quasilinear elliptic problems, Differential Integral Equations 7 (1994), 1453-1471.

[20] F. Mignot, F. Murat, and J. P. Puel, Variation d'un point de retournement par rapport au domaine, Comm. Partial Differential Equations 4 (1979), 1263-1297.

[21] K. Nagasaki and T. Suzuki, Asymptotic analysis for two-dimensional elliptic eigenvalue problems with exponentially dominated nonlinearities, Asymptotic Analysis 3 (1990), 173188.

[22] K. Nagasaki and T. Suzuki, Radial solutions for $\Delta u+\lambda e^{u}=0$ on annuli in higher demensions, J. Differential Equations 100 (1992), 137-161.

[23] K. Nagasaki and T. Suzuki, Spectral and related properties about the Emden-Fowler equation $-\Delta u=\lambda e^{u}$ on circular domains, Math. Ann. 299 (1994), 1-15.

[24] S. I. Pohozaev, Eigenfunctions of the equation $\Delta u+\lambda f(u)=0$, Soviet Math. Dokl. 6 (1965), 1408-1411.

[25] P. H. Rabinowitz, Some global results for nonlinear eigenvalue problems, J. Func. Anal. 7 (1971), 487-513.

[26] P. H. Rabinowitz, Some aspects of nonlinear eigenvalue problems, Rocky Mountain J. Math. 3 (1973), 161-202.

[27] T. Senba and T. Suzuki, Some structures of the solution set for a stationary system of chemotaxis, Adv. Math. Sci. Appl. 10 (2000), 191-224.

[28] T. Suzuki, Global analysis for a two-dimensional elliptic eigenvalue problem with the exponential nonlinearity, Ann. Inst. Henri Poincaré Anal. Non Linéaire 9 (1992), 367-398. 
[29] T. Suzuki, Energy transport problem for self-gravitating particles, Taiwanese J. Math., to appear.

[30] T. Suzuki, Semilinear Elliptic Equations, Gakkōtosho, Tokyo, 1994.

[31] T. Suzuki, Free Energy and Self-Interacting Particles, to be published by Birkhäuser, Boston.

[32] G. Tarantello, Multiple condensate solutions for the Chern-Simons-Higgs theory, J. Math. Phys. 37 (1996), 3769-3796.

[33] G. Wolansky, On the evolution of self-interacting clusters and applications to semilinear equations with exponential nonlinearity, J. Anal. Math. 59 (1992), 251-272.

[34] Y. Yang, Solitons in Field Theory and Nonlinear Analysis, Springer, New York, 2001. 